\title{
Elemental Analysis of Cells and Tissues
}

\author{
Peta L. Clode ${ }^{1}$ \\ ${ }^{1}$ Centre for Microscopy, Characterisation \& Analysis, The University of Western Australia. Crawley, \\ WA 6009 Australia.
}

Elemental and isotopic imaging and analysis of cells and tissues is commonly utilised in the plant and animal sciences, being driven by scientific questions relating to disease states, resistance or susceptibility of different genotypes to environmental conditions, the role of partners in symbioses, biomineral formation, and cellular function and dynamics [1-4]. There are numerous analytical microscopy-based methods available - each with their advantages and limitations - and the method(s) employed must be tailored to the question at hand and thus be able to address the specific requirements relating to analytical resolution (e.g. subcellular, single cells, cell layers), sensitivity (low or high concentrations), elements of interest, and the forms of data (e.g. quantifiable, concentrations, maps/spectra) that are required.

Available imaging-based techniques include energy-filtered TEM (EFTEM), electron probe x-ray microanalysis (EDS), x-ray nanoprobe analysis, and secondary ion mass spectrometry (SIMS). This plenary talk however, will aim to give an overview of the application of some electron (e.g. EDS and EFTEM) and ion (e.g. NanoSIMS) based analytical systems to the elemental and isotopic analysis of cells and tissues in biology. Examples will extend from toxicity (e.g. salinity, phosphorus and calcium) and metals (e.g. hyperaccumulation, gold nanoparticle formation) in plants, to iron- and calcium-based biomineralising tissues, iron-based magnetoreceptors, nutrient dynamics in plant-microbial-soil environments, and symbiotic algal cells.

Focus will be given to the reasons one might consider adopting one or more of these methods, and to the limitations that can be expected. Factors to consider when using different methods of sample preparation, including chemical fixation and dehydration, low temperature fixation, freeze-substitution and drying, and frozen-hydrated preparations, will be outlined. Additionally, practical challenges that biologists regularly face around sampling and sample preparation, element retention and immobilisation, experimental replication, low concentrations and detection limits, and analytical support will be touched upon.

Additional examples and alternative methods of analysis that are highly suited to the elemental analysis of biological materials will also be presented by invited speakers and contributed talks as part of the Microanalysis of Biological Materials Symposium at the Microscopy and Microanalysis meeting that follows IUMAS [5].

\section{References:}

[1] P Echlin, Microscopy and Microanalysis 7, 211-219 (2001).

[2] M McCully, M Canny, C Huang, C Miller \& F Brink, Func. Plant Bio. 37, 1011-1040 (2010)

[3] M Pernice et al. ISME J 1-11 (2012).

[4] A Marshall, P Clode, R Russell, K Prince \& R Stern, J. of Exp. Biology 210, 2453-2463 (2007). 
[5] The author acknowledges ARC Discovery Program funding (DP130100005) and the use of the equipment at CMCA, a facility funded by Universities, and State and Commonwealth governments.
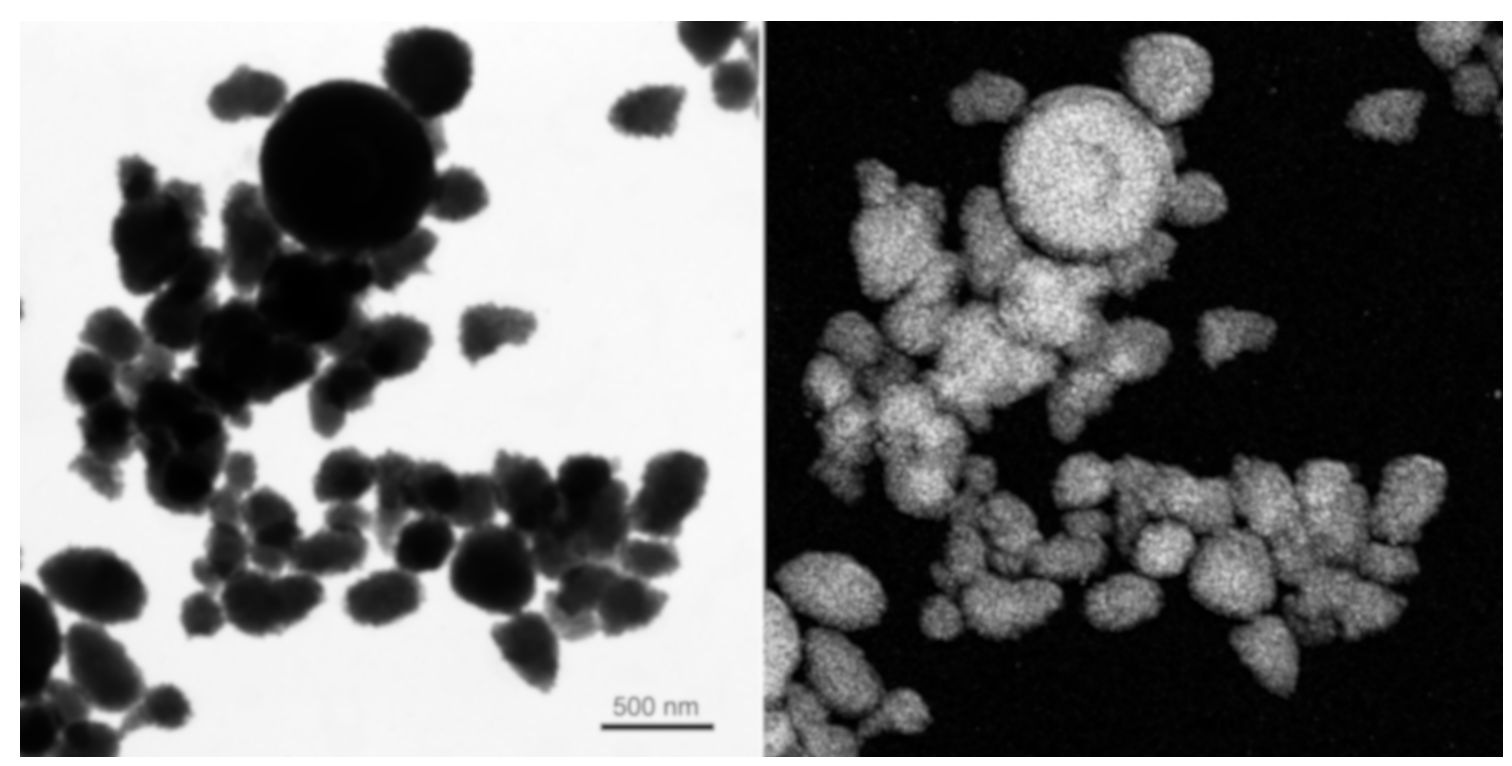

Figure 1: TEM brightfield image of granules extracted from trophocyte cells of the honey bee Apis mellifera

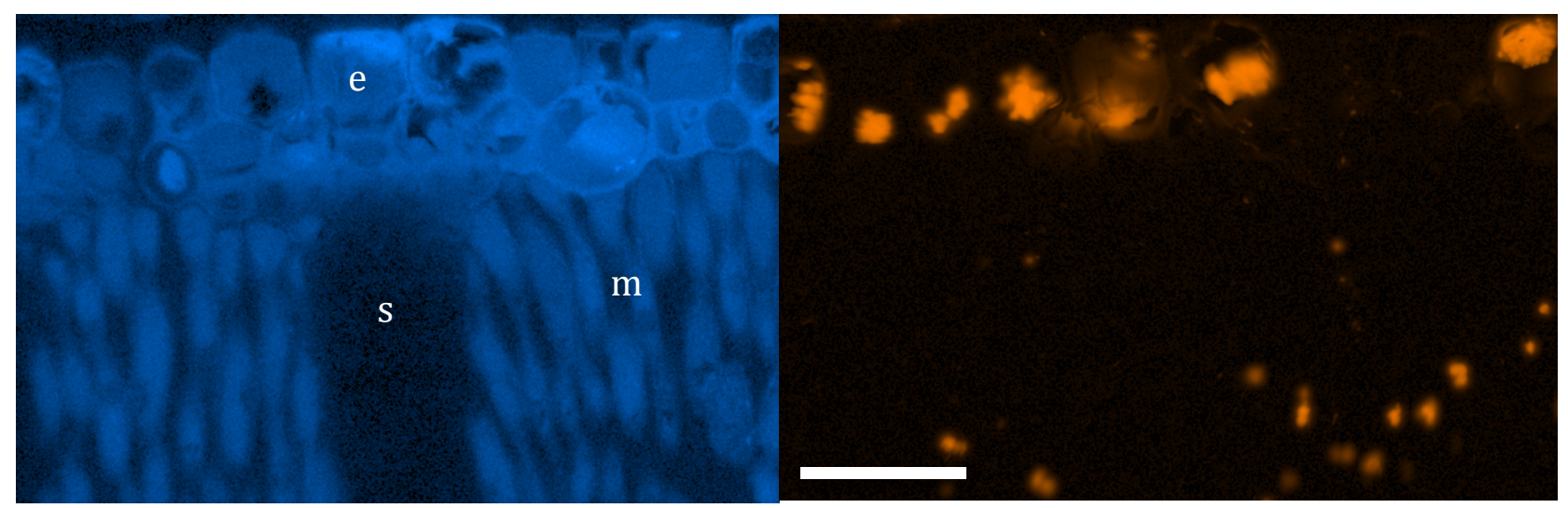

Figure 2: EDS maps from transverse sections of freeze-substituted Banksia attenuata leaves, showing chloride (left) and silicon (right) distribution in the epidermis (e), sclerenchyma (s), and mesophyll (m). Scale bar $=50 \mathrm{um}$. 